\title{
The Research of Power Quality Prediction and Evaluation Method for the Large-scale Charging Load
}

\author{
Tianyi Qu ${ }^{1, a}$ and Kui Chen ${ }^{2, b}$ \\ ${ }^{1}$ School of Management, XuZhou institute of Technology, Xuzhou, Jiangsu, China 221008 \\ ${ }^{2}$ China University of Mining \& Technology, Xuzhou, Jiangsu, China 221008 \\ ajdbh2001@163.com bckbhxtlc73@sina.com
}

Keywords: Electric vehicle; Charging load; Power quality; Prediction; Evaluation

\begin{abstract}
Combined with the project of the development guidelines for charging infrastructure of the electric car (2015-2016) and the plan of Distribution network's construction and renovation action (2015-2020), this article is predicted and evaluated to power quality of the large-scale electric vehicle total charging load with the energy gravity method and classification disturbance method. The results of prediction and evaluation has the good reference value to research and plan the charging load accessing and related measures of the large-scale electric vehicle.
\end{abstract}

\section{Introduction}

Increasing development of electric vehicle can accelerate alternative fuel, reduce emissions from cars, and have important meaning to ensure the energy safety, prevent and control atmospheric pollution, and promote energy saving and emission reduction and our country from a big country to a powerful country in automobile industry.

According to the plan of The Development Guidelines for Charging Infrastructure of The Electric Car(2015-2016)and the requirement of The Plan of Distribution Network's Construction and Renovation Action(2015-2020), the power department should achieve the connection between the distribution network planning and charging electric facility planning, strengthen the construction and reform of the charging electric facilities that matched with power network, and guarantee the barrier-free access of charging electric facilities. They also should accelerate build the intelligent service network for electric vehicle charging, promote the operation technology of the electric vehicle charging orderly, V2G,as well as charging storage integration, and achieve the interconnectivity of charging facilities between cities and inter-cities. By 2020, 12 thousand charging electric station will have been satisfied, and 4.8 millions charging piles will have been connected, which provides charging electric service for 5 millions electric vehicles. Because most of the charging infrastructure consist of power electronic components, or it will cause some interferences , and the large-scale planning as well as rapid development of charging electric facilities accessing will affect the power quality of distribution network. Therefore carrying out the detection and evaluation to large-scale charging load power quality not only has important meaning to ensure the power network safe, reliable and highly efficient operation, also promote the .

\section{A Review of Domestic and Foreign Research}

Started relatively late compared with the developed countries, electric cars in China has fast development. To meet the needs of the electric car development of infrastructure, the provinces and energy companies are promoting the building of charging infrastructure. In April 2010, issuing four national standards, namely The Experiment Method to The Light Hybrid Electric Vehicle Energy Consumption Amount, The General Requirements for Electric Vehicle Charging Station, The Communication Protocol between The Management System of The electric Vehicle Battery and Off-board Charger, and The Charging Interface of Electric Cars Conduction Type, the nation will implement the subsidy and supporting policies to new energy vehicles, such as the electric cars. At 
present, the electric vehicle charging infrastructure has entered a rapid large-scale implementation stage, and some running charging infrastructure has been affecting continuously to the power grid voltage, unbalance, loss, sag, voltage flicker and harmonic, and so on.

The power quality influence of power system by large-scale charging stations mainly reflects in harmonic, inter-harmonic and sag to increase power energy disturbance, etc. Therefore, many domestic researchers research the problem of harmonic pollution to power grid through modeling and simulation, and compare a common analysis method by setting up the simulation model on the stage of Matlab Simulink, and research the factors of affecting the size of charging infrastructure harmonic though measurement and proof validation. Or we can use collected data from commercial electric car charger to calculate the harmonic current, set up harmonic analysis mathematical model with multiple harmonic sources by using probability and statistics law of large Numbers and central limit theorem, and then research the harmonic current and probability characteristic with multiple electric car chargers causing. Some scholars propose the method of predicting the total harmonic current which produced by a set of electric vehicle charging machine. This method considers randomness that single charger starts charging time and battery's remaining power, and use random theory to model. Used the model, the total harmonic currents of a group of chargers producing not only can be simulated and analyzed, but also the influence that chargers affect transformers and the supply network voltage harmonic. Other scholars study that single-phase charging machine has an effect the supply network total current harmonic distortion rate, total voltage harmonic distortion rate, as well as transformer performance. They also recommend that charger is charged during slack time at night to reduce the impact on the supply network and low the investment of power system.

In addition, the power pollution problem of the distribution network rarely is researched deeply to the large-scale electric vehicles charging load assess in domestic. And the present studies are limited to the influence that integral harmonics to the power grid .And there are few researches and analysis to flicker and inter-harmonics. So there exists a problem that researching contents are incomplete and the optimization strategies are not enough deep.

\section{The Prediction and Evaluation to the Scale Charging Load Power Quality}

On the analysis of simulation, fully considering the diversity of power system, the author widely simulates a variety of connection mode to the power grid. When the charging load is modeled, it should be considered that the various types of charging infrastructure((now there are mainly three phase bridge rectifier charger, 12 pulse rectifier charger, APF compensation rectifier charger, PWM rectifier charger), and all kinds of charging ways(mainly slow filling, quick charge, super capacitor charging, etc ). It should be through a comprehensive simulation obtained that the specific characteristics and size of electric energy disturbance such as harmonics, transient voltage drop, flicker, etc by the various the electric car charging infrastructure producing. On this basis, the relevant properties of many charging facilities are simulated and analyzed in different access time, and different the electric car initial capacity, etc.

In the analysis and calculation, the multiple frequency electric power harmonic signal is set, which can be expressed as follows:

$$
x(t)=\sum_{p=1}^{P} A_{p} \cos \left(2 \pi r_{p} f_{1} t+\varphi_{p}\right)
$$

In the formula, $\mathrm{p}$ is the sequence number. $\mathrm{P}$ is the total number of harmonic component. ${ }^{r}$ is harmonic number, when the $r_{p}$ is positive integer, the given signal is harmonic, and when a decimal, then harmonics. $A_{p}$ is the $r_{p}$ times harmonic amplitude. $f_{1}$ is the fundamental wave angular frequency of power signal . $\varphi_{p}$ is the $r_{p}$ times harmonic beginning phase angle.

The equation (1) is numerical sequence after is scattered 


$$
x(n)=\sum_{p=1}^{P} A_{p} \cos \left(2 \pi r_{p} f_{1} T_{s} n+\varphi_{p}\right)
$$

In the formula, Ts is sample period. $\mathrm{n}$ is serial number, $\mathrm{n}=1,2, \ldots, \mathrm{W}(\mathrm{n})$ weighted $\mathrm{x}(\mathrm{n})$, which is a symmetric cosine window function

$$
\begin{aligned}
& x_{w}(n)=x(n) \cdot w(n) \\
& w(n)=\sum_{i=0}^{m}(-1)^{i} a_{i} \cos \left(\frac{2 \pi i}{N} n\right)
\end{aligned}
$$

Among the above formula, $\sum_{i=0}^{m} a_{i}=1, \sum_{i=0}^{m}(-1)^{i} a_{i}=0, \quad \mathrm{n}=0,1, \ldots, \mathrm{N}-1, \mathrm{~N}$ is the sequence length of $\mathrm{W}$ (n). $m+1$ is the number of window function.

If $k_{p m}$ is integer, that is to say the time NTs is integer multiple of the analyzed signal period, then $k_{p}=k_{p m}$ is exactly a FFT spectrum value of the sequence spectrum ( ${ }^{k_{p}}$ is the maximum spectral line of the $r_{p}$ times harmonic or inter harmonic), and located in the center of window function. Therefore $X_{w}\left(k_{p}\right)=A_{p} e^{j \varphi_{p}}$. However, due to the non synchronous sampling, or analyzed signal with non-integral harmonic, ${ }^{k_{p m}}$ generally is not integer times of NTS, means $k_{p}$ is not equal to $k_{p m}$, which is not in the gravity of the window function and deviating from $\Delta k_{p}$. It is can be proved that when any one of the symmetrical cosine window function is given and $\mathrm{q}$ is large enough, then the equation (5) is existence.

$$
k_{p m}=\sum_{k=-q}^{q} k W^{2}\left(k-k_{p m}\right) / \sum_{k=-q}^{q} W^{2}\left(k-k_{p m}\right)=\sum_{k=-q}^{q} k X_{w}^{2}(k) / \sum_{k=-q}^{q} X_{w}^{2}(k)
$$

We use the equation (6) and the equation (7) to estimate the power harmonic frequency, and use the equation (8) and the equation (10) to respectively estimate the phase and amplitude of each electric power harmonic. Because the parameters of each power harmonic is estimated with the main lobe power, the proportion of main lobe power is higher than the total power, and estimation precision of the power harmonic parameters is higher. The main lobe width of the symmetrical cosine window function is $2(m+1) \Delta f$, and then the equation (5) is approximated as follows:

$$
k_{p m}= \begin{cases}\sum_{k=k_{p}-m}^{k_{p}+m+1} k X_{w}^{2}(k) / / \sum_{k=k_{p}-m}^{k_{p}+m+1} X_{w}^{2}(k) & X\left(k_{p}+1\right) \geq X\left(k_{p}-1\right) \\ \sum_{k=k_{p}-m-1}^{k_{p}+m} k X_{w}^{2}(k) / \sum_{k=k_{p}-m-1}^{k_{p}+m} X_{w}^{2}(k) & X\left(k_{p}-1\right) \geq X\left(k_{p}+1\right)\end{cases}
$$

In the formula, $k_{p}$ is max spectral line of $r_{p}$ times harmonics or inter- harmonics.

$$
r_{p} f_{1}=k_{p m} \Delta f=k_{p m} / T_{S} N
$$

From the equation (6), we can get $\varphi_{k p}=\varphi_{p}-\pi \Delta k_{p}$, and phase correction values of each harmonic

$$
\varphi_{p}=\varphi_{k p}+\pi \Delta k_{p}=\varphi_{k p}+\pi\left(k_{p}-k_{p m}\right)
$$

Among them, $\varphi_{k p}$ is the phase of $r_{p}$ times peak harmonic spectral lines.

On the basis of Parseval's theorem, the frequency spectrum of the signal power should equal the total power of signal in time domain. 


$$
\left\{\begin{aligned}
\frac{1}{N} \sum_{n=0}^{N-1}[x(n) \cdot w(n)]^{2} & =\sum_{k=-(N-1) / 2}^{(N-1) / 2} X_{w}^{2}(k)=X_{w}^{2}\left(k_{p m}\right) \\
\frac{1}{N} \sum_{n=0}^{N-1} x^{2}(n) & =\sum_{k=-(N-1) / 2}^{(N-1) / 2} X^{2}(k)=A_{p}^{2}
\end{aligned}\right.
$$

$X(k)$ is the FFT value of sequence $\mathrm{x}$ (n) that has the same length with the window function w (n), means $X(k)=F F T[x(n)]$.In frequency domain, main lobe power is approximately seen as total power:

$$
A_{p}^{\prime}=\left\{\begin{array}{lc}
\sqrt{K_{g} \sum_{k=k_{p}-m}^{k_{p}+m+1} X_{w}^{2}(k)}, & X\left(k_{p}+1\right) \geq X\left(k_{p}-1\right) \\
\sqrt{K_{g} \sum_{k=k_{p}-m-1}^{k_{p}+m} X_{w}^{2}(k),} & X\left(k_{p}-1\right)>X\left(k_{p}+1\right)
\end{array}\right.
$$

Among the above equation, $A_{p}^{\prime}$ is the $r_{p}$ times harmonic estimates. $K_{g}$ is the power recovery coefficient of the window function.

$$
\begin{aligned}
& K_{g}=\frac{1}{N} \sum_{n=0}^{N-1} x^{2}(n) / \frac{1}{N} \sum_{n=0}^{N-1}[w(n) x(n)]^{2} \\
& \text { If }^{x(n)=1}, \text { then } \\
& K_{g}=N / \sum_{n=0}^{N-1} w^{2}(n)
\end{aligned}
$$

In this way, we can estimate this complex harmonic amplitude, frequency and phase angle with the energy gravity method. Meanwhile we can identify flicker and sag based on the power quality disturbance classification method in time domain.

\section{Conclusion}

On the basis of analyzing and measuring the probable generated harmonics, sag, flicker and a variety of disturbance of the electric vehicle charging facilities, the relevant influence is evaluated in detail with the data envelopment analysis method, especially the relative merits between multiple similar sample are evaluated. This project pay attention to the influence of harmonic to flicker, therefore it is provided firstly that the various disturbance index of before and after connecting large-scale charging load by using EDA. Then the comprehensive evaluation system is established to weight each points indicator. It is evaluated that whether appropriately access high-capacity charging load to access point with the evaluation results, and the installed and management advice are given.

\section{References}

[1] Owen Worley, Diego KIabjall. Optimization of battery charging and purchasing at electric vehicle battery swap--Stations[C], vehicle Power and Propulsion Conference (vppc), 2011 IEEE, 20M; 1--4.

[2] Wang Zhenpo, Liu Peng, Xin Tao. Optimizing the quantity of Off-broad charger for whole vehicle charging station [C], 2010 International Conference on Optoelectronics and Image Processing(ICOIP), 2010; 93--96.

[3] Thoralf Winkler, Przemyslaw Komarnieki, Gerhard Mud--ler, Electric vehicle charging stations in Magdeburg [c],Vehicle Power and Propulsion Conference(VPPC), 2009,IEEE, 2009; 60--65.

[4] Deng Benzai, Wang Zhiqiang, Research on electric-vehicle charging station technologies based on smart grid[C],Asia-Pacific Power and Energy Engineering Conference(APPEEC), 2011; $1--4$. 
[5] LI Xiaoqiang. Research and design of PEV batteries charging and exchanged station simulation software based on maximum load [D]. Beijing; Beijing Jiaotong University, 2011.

[6] Clement-Nyns, KHaesen E, Driesen J. The impact of charging plug--in hybrid electric vehicles on a residential distribution grid [J]. IEEE Transactions on Power Systems, 2010, 25(1); 371-380.

[7] Putrus G A, Suwanapingkarl P, Johnston P, et a1.Impact of electric vehicles on power distribution networks[C], IEEE Vehicle Power and Propulsion Conference. Dearborn, USA; IEEE, 2009; 827. 831. 